\title{
Brucellosis Manifesting as Chronic Inflammatory Demyelinating Polyneuropathy
}

\author{
Bong-Hui Kang, Young-Min Lim, Kwang-Kuk Kim
}

Can J Neurol Sci. 2012; 39: 536-538

Brucellosis is a zoonotic infection transmitted to humans after ingestion of contaminated foods or direct contact with infected animal carcasses or placentas. It is a multisystem disorder that can involve any organ including the central and peripheral nervous system. Neurobrucellosis is mainly due to the direct effects of intracellular Brucella organisms ${ }^{1}$, but an immune-mediated mechanism may be associated with pathological processes ${ }^{2}$. We describe here a patient with neurobrucellosis manifesting as chronic inflammatory demyelinating polyradiculoneuropathy and associated with an immune mechanism triggered by Brucella infection.

\section{CASE}

A 28-year-old man presented to the Department of Neurology at the Asan Medical Center with a subacute onset of progressive weakness in both legs. Two months before admission, he had developed back pain and weakness and tingling of his distal legs.
Subsequently, he noted facial muscle weakness and difficulty walking. Two months prior to symptom onset, he had delivered a dead calf from a cow, later found to be infected with Brucella. He was tested for brucellosis at a community health center one week before admission. He had no medical history and no family history of neuromuscular disease. Neurological examination showed facial diplegia, symmetric flaccid weakness [Medical Research Council (MRC) grade 3] and complete vibration loss in his distal legs with muscle atrophy and areflexia in all extremities. His thyroid function and serum glucose concentration were normal. He was serologically negative for syphilis, collagen vascular disease, and human immunodeficiency virus (HIV) infection. Immunofixation electrophoresis showed no abnormal proteins in his serum or urine. His serum was positive for IgM anti-GM1 antibodies, with a titer of $35.3 \mathrm{EU} / \mathrm{mL}$ (reference value: < $20 \mathrm{EU} / \mathrm{mL}$ ). Motor nerve conduction studies (NCS) showed markedly delayed distal

Table: Motor nerve conduction study

\begin{tabular}{|c|c|c|c|c|c|c|c|c|c|c|c|}
\hline \multirow[b]{2}{*}{ Nerve } & \multirow[b]{2}{*}{$\begin{array}{c}\text { Stimulation } \\
\text { site }\end{array}$} & \multirow[b]{2}{*}{$\begin{array}{c}\text { Recording } \\
\text { site }\end{array}$} & \multicolumn{5}{|c|}{ Baseline } & \multicolumn{4}{|c|}{ 2-months follow-up } \\
\hline & & & & $\begin{array}{l}\text { Latency } \\
\text { (ms) }\end{array}$ & $\begin{array}{l}\text { Amplitude } \\
(\mathrm{mV})\end{array}$ & $\begin{array}{c}\text { CV } \\
(\mathrm{m} / \mathrm{s})\end{array}$ & $\begin{array}{l}\text { F-w } \\
\text { (ms) }\end{array}$ & $\begin{array}{l}\text { Latency } \\
\text { (ms) }\end{array}$ & $\begin{array}{c}\text { Amplitude } \\
(\mathrm{mV})\end{array}$ & $\begin{array}{c}\text { CV } \\
(\mathrm{m} / \mathrm{s})\end{array}$ & $\begin{array}{l}\text { F-w } \\
\text { (ms) }\end{array}$ \\
\hline \multirow[t]{2}{*}{ Median } & Wrist & APB & $L$ & 9.0 & 11.3 & 42.5 & 52.0 & 9.4 & 10.6 & 43.3 & 50.3 \\
\hline & & & $\mathrm{R}$ & 8.8 & 14.5 & 36.5 & 54.3 & 8.8 & 11.9 & 39.3 & 54.1 \\
\hline \multirow[t]{2}{*}{ Ulnar } & Wrist & ADM & $L$ & 6.0 & 11.4 & 34.7 & 54.2 & 6.6 & 11.2 & 35.6 & 57.3 \\
\hline & & & $\mathrm{R}$ & 5.8 & 10.8 & 33.8 & 49.3 & 6.0 & 11.9 & 31.8 & 49.9 \\
\hline \multirow[t]{2}{*}{ Peroneal } & Anterior & EDB & $L$ & 13.2 & 0.2 & 28.7 & $N R$ & 16.0 & 0.2 & 18.9 & $N R$ \\
\hline & ankle & & $\mathrm{R}$ & 13.4 & 0.5 & 33.3 & $N R$ & 18.0 & 0.2 & 23.2 & $N R$ \\
\hline \multirow[t]{2}{*}{ Tibial } & Medial & $\mathrm{AHB}$ & $\mathrm{L}$ & 8.1 & 0.3 & 34.0 & $N R$ & 12.7 & 0.1 & 21.1 & $N R$ \\
\hline & ankle & & $\mathrm{R}$ & 7.6 & 0.1 & 22.5 & $N R$ & & NR & & $N R$ \\
\hline
\end{tabular}

$\mathrm{CV}$; conduction velocity, F-w; F-wave latency, APB; abductor pollicis brevis, ADM; abductor digiti minimi, EDB; extensor digitorum brevis, AHB; abductor hallucis brevis, L; left, R; right, NR; no response, Boldface values indicate demyelinating features by electrodiagnostic criteria of CIDP. 


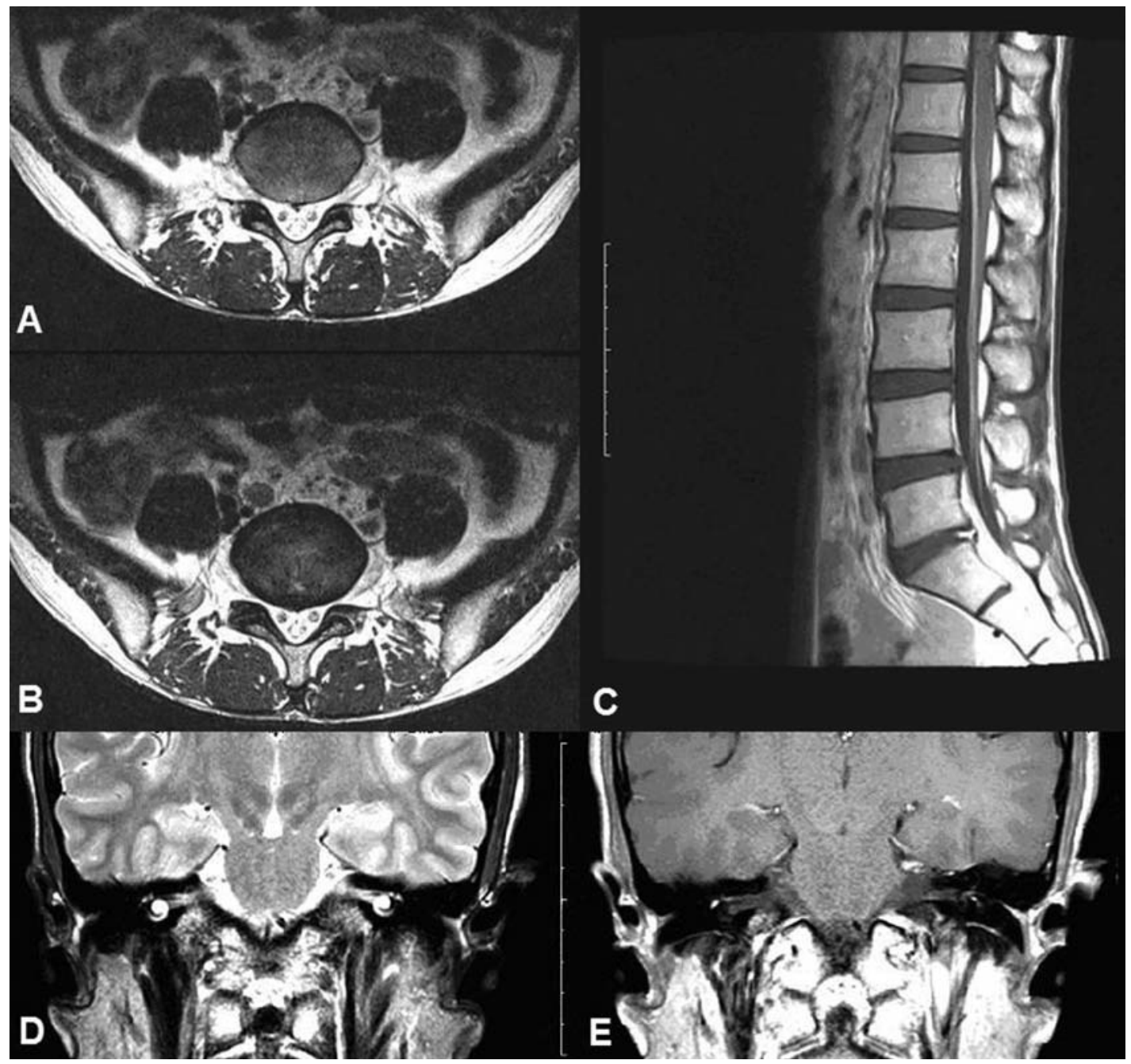

Figure: Lumbar spine and brain MRI. (A and B) The T2-weighted axial images show hypertrophy of dorsal root ganglions and lumbar spinal nerve roots. (C) The contrast-enhanced T1-weighted sagittal image shows enhancement of lumbar spinal nerve roots. (D) The bilateral facial nerves show thickening and high signal intensity on the T2weighted image. (E) The contrast-enhanced T1-weighted image shows bilateral mild enhancement of the facial nerves.

latencies and slow conduction velocities in all nerves, decreased amplitudes of compound muscle action potentials in his legs, prolonged latency of F-waves in his arms, and an absence of Fwaves in his legs, all of which were compatible with demyelinating polyneuropathy (Table). Sensory NCS showed an absent superficial peroneal sensory response and decreased amplitude of the distal part of the median and ulnar nerves with slow conduction velocity. Cerebrospinal fluid (CSF) analysis showed albumino-cytologic dissociation; white blood cells $2 / \mathrm{mm}^{3}$, red blood cells $3 / \mathrm{mm}^{3}$, protein $370.4 \mathrm{mg} / \mathrm{dL}$ (reference value: $15-45$ ) and glucose $3.9 \mathrm{nmol} / \mathrm{L}$. Lumbar spine magnetic resonance imaging (MRI) showed enhancement and hypertrophy of the dorsal root ganglions and nerve roots (Figure A, B and C). Brain MRI showed thickening and T2 signal increase with mild enhancement of both facial nerves (Figure D and E). He was diagnosed with chronic inflammatory demyelinating polyradiculoneuropathy (CIDP) and treated with steroid pulse therapy, followed by oral steroid maintenance.
Immediately after discharge, he was informed by the public health center that he was positive for anti-brucella antibody, with an agglutination titer of 1:20. A second test four weeks later showed a four-fold increase in agglutinating antibodies, to a titer of 1:80. Promptly after diagnosis of brucellosis, doxycycline (200mg/day) and rifampin (600mg/day) were given along with steroids. However, his paraparesis deteriorated (MRC grade 2/5) continuously and he was not able to walk without help. Also he developed weakness in his distal arms (MRC grade 4/5). He was hospitalized again 45 days after discharge. Follow-up IgM antiGM1 antibody was positive in his serum, with a titer of 33.0 $\mathrm{EU} / \mathrm{mL}$. His CSF and blood cultures were negative. Follow-up NCS showed slightly worsened findings since the previous examination (Table). Electromyography showed evidence of active denervation in his extremities, paraspinal and facial muscles. He was started on intravenous immunoglobulin $(0.4$ $\mathrm{gm} / \mathrm{kg} /$ day for five days) along with oral steroid and the antibrucella antibiotics including rifampin, doxycycline, and 
trimethoprim/sulfamethoxazole. At the sixth month of treatment, his facial diplegia markedly improved. His leg muscle strength recovered to MRC grade 4/5 and he was able to walk unaided.

\section{Discussion}

Our patient presented with a CIDP associated with brucellosis. He had a chronic progressive course of facial diplegia, areflexic symmetric weakness and sensory loss in both legs for about four months. Electrodiagnostic studies revealed demyelinating sensorimotor polyneuropathy, and facial NCS also showed demyelinating features. CSF analysis demonstrated albumino-cytologic dissociation. Brain and spine MRI showed hypertrophy and enhancement of lumbar spinal roots and facial nerves. His clinical and electrophysiologic findings were compatible with the criteria of $\mathrm{CIDP}^{3}$. He had been exposed to the contaminated placenta of his cow two months before onset of his neurological symptoms. Although he did not have acute fever and Brucella species were not isolated from his clinical specimens, the Brucella agglutination titer in his serum increased four-fold over four weeks. His exposure history and serologic results provided strong evidence for a diagnosis of brucellosis. Moreover, thorough laboratory examination excluded other diseases associated with CIDP, including monoclonal gammopathy, HIV infection, connective tissue disease, diabetes, and thyroid disease.

The nervous system is affected in $3-5 \%$ of patients with brucellosis, with various and complex symptoms ${ }^{1,4}$. Acute meningoencephalitis is the most common manifestation of neurobrucellosis. Other clinical categories include chronic meningitis, meningovascular complications, myelitis, cranial nerve involvement, and polyradiculoneuropathy. Proximal polyradiculoneuropathy is the main peripheral form of neurobrucellosis ${ }^{4}$. It is clinically characterized by areflexic paraparesis with normal sensation and CSF lymphocytic pleocytosis, resembling Guillain-Barre syndrome (GBS). Moreover, there was a report of acute axonal form of GBS associated with partially treated brucellosis ${ }^{2}$. The distinguishing features of our case relative to those already published were chronic disease course of inflammatory demyelinating neuropathy and CSF albumin-cytologic dissociation, compatible with CIDP. To our knowledge, this is the first case report presenting with CIDP triggered by brucellar infection.

A recent animal study showed that a lipooligosaccharide of Brucella melitensis had a GM1 ganglioside-like structure and induced a strong antibody response in mice with GBS-like symptoms $^{5}$. A molecular mimicry between Brucella lipooligosaccharide and GM1 ganglioside may be one of the autoimmune mechanisms leading to polyradiculoneuropathy associated with brucellosis. In our patient, anti-GM1 antibody was positive and his neurologic deficits improved after immunomodulatory therapy and treatment with anti-brucella antibiotics.

In conclusion, our case demonstrates that brucellosis can be associated with CIDP as triggering an autoimmune response. Brucellar infection should be considered in patients with CIDP for proper management, especially if they have history of exposure to infected animals even in the absence of other systemic signs.

\section{REFERENCES}

1. al Deeb SM, Yaqub BA, Sharif HS, Phadke JG. Neurobrucellosis: clinical characteristics, diagnosis, and outcome. Neurology. 1989;39(4):498-501.

2. Haghighi AB, Sabayan B. Acute axonal poly-radiculoneuropathy associated with partially treated brucellosis: a case report. J Clin Neuromuscul Dis. 2007;9(1):262-4.

3. Hughes RA, Bouche P, Cornblath DR, et al. European Federation of Neurological Societies/Peripheral Nerve Society guideline on management of chronic inflammatory demyelinating polyradiculoneuropathy: report of a joint task force of the European Federation of Neurological Societies and the Peripheral Nerve Society. Eur J Neurol. 2006;13(4):326-32.

4. Shakir RA, Al-Din AS, Araj GF, Lulu AR, Mousa AR, Saadah MA. Clinical categories of neurobrucellosis. A report on 19 cases. Brain. 1987;110(Pt 1):213-23.

5. Watanabe K, Kim S, Nishiguchi M, Suzuki H, Watarai M. Brucella melitensis infection associated with Guillain-Barre syndrome through molecular mimicry of host structures. FEMS Immunol Med Microbiol. 2005;45(2):121-7. 